\title{
LIST OF PARTICIPANTS
}

Abramowicz, M. A., Zaklad Astronomii PAN, Warszawa, Poland

Aichelburg, P. C., Universität Wien, Wien, Austria

de Angelis, U., Osservatorio Astronomico Capodimonte, Napoli, Italy

Arnett, W. D., Rice University, Houston, U.S.A.

Balkowski, C., Observatoire de Paris, Meudon, France

Bardeen, J. M., Yale University, New Haven, U.S.A.

Basko, M. M., Institute of Applied Mathematics, Moscow, U.S.S.R.

Bergmann, P. G., Syracuse University, Syracuse, U.S.A.

Bertotti, B., Universita di Pavia, Pavia, Italy

Bičak, J., Charles University, Praha, Czechoslovakia

Bludman, S. A., University of Pennsylvania, Philadelphia, U.S.A.

Bonazzola, S., Observatoire de Paris, Meudon, France

Borzeszkowski, H. H., Zentralinstitut für Astrophysik, Potsdam-Babelsberg, G.D.R.

Boynton, P., University of Washington, Seattle, U.S.A.

Braginsky, V. B., Moscow State University, Moscow, U.S.S.R.

Burke, W. L., Lick Observatory, Santa Cruz, U.S.A.

Carr, B. J., University of Cambridge, Cambridge, England

Carson, T. R., University Observatory, Fife, England

Carter, B., University of Cambridge, Cambridge, England

Chandrasekhar, S., University of Chicago, Chicago, U.S.A.

Chevalier, C., Saint-Michel - l'Observatoire, Forcalquier, France

Choquet-Bruhat, Y., Université de Paris, Paris, France

Chrzanowski, P., University of Maryland, College Park, U.S.A.

Chubaryan, E. V., Yerevan State University, Yerevan, U.S.S.R.

O'Connell, R. F., Louisiana State University, Baton Rouge, U.S.A.

Contopoulos, G., Thessaloniki University, Thessaloniki, Greece

Demiański, M., Uniwersytet Warszawski, Warszawa, Poland

DeWitt-Morette, C., University of Texas, Austin, U.S.A.

Doroshkievich, A. G., Institute of Applied Mathematics, Moscow, U.S.S.R.

Doughty, N. A., University of Canterbury, Christchurch, New Zealand

Douglas, D. H., University of Rochester, Rochester, U.S.A.

Drever, R. W. P., University of Glasgow, Scotland

Ehlers, J., Max-Planck-Institut, München, F.R.G.

Fairbank, W. M., Stanford University, Stanford, U.S.A.

Faulkner, J., Lick Observatory, Santa Cruz, U.S.A.

Field, G. B., Harvard College Observatory, Cambridge, U.S.A.

Fishbone, L. G., University of Utah, Salt Lake City, U.S.A. 
Forlani, A., Osservatorio Astronomico Capodimonte, Napoli, Italy Fowler, W. A., California Institute of Technology, Pasadena, U.S.A. Friedman, J. L., Yale University, New Haven, U.S.A.

Ganea, I. M., Astronomical Observatory, Bucharest, Rumania Giacconi, R., American Science and Engineering, Cambridge, U.S.A. Gibbons, G. W., University of Cambridge, Cambridge, England Godart, O., Université Catholique de Louvain, Louvain, Belgium Goldberg, L., Kitt Peak National Observatory, Tucson, U.S.A. Grishchuk, L. P., Sternberg Astronomical Institute, Moscow, U.S.S.R. Hamilton, W. O., Louisiana State University, Baton Rouge, U.S.A. Hartle, J. B., University of California, Santa Barbara, U.S.A. Hawking, S. W., University of Cambridge, Cambridge, England Heintzmann, H., Universität zu Köln, Köln, F.R.G. von Hoerner, S., National Radio Observatory, Green Bank, U.S.A. Imshennik, V. S., Institute of Applied Mathematics, Moscow, U.S.S.R. Isaacson, R., Illinois Institute of Technology, Chicago, U.S.A. Ivanenko, D. D., Moscow State University, Moscow, U.S.S.R. Jaffe, J., Smithsonian Astrophysical Observatory, Cambridge, U.S.A. John, R. W., Zentralinstitut für Astrophysik, Potsdam-Babelsberg, G.D.R. Kafka, P., Max-Planck-Institut, München, F.R.G.

Kampfer, B., Friedrich-Schiller-Universität, Jena, G.D.R. Kardashev, N. S., Sternberg Astronomical Institute, Moscow, U.S.S.R. Kraft, R. P., Lick Observatory, Santa Cruz, U.S.A.

Kreisel, B., Zentralinstitut für Astrophysik, Potsdam-Babelsberg, G.D.R.

Kundt, W., Universität Bielefeld, Bielefeld, F.R.G.

Langer, J., Charles University, Prague, Czechoslovakia Lasota, J. P., Zakład Astronomii PAN, Warszawa, Poland

Logan, J. L., Rockefeller University, New York, U.S.A.

Lund, F., University Observatory, Oxford, England MacCallum, M. A. H., University of Cambridge, Cambridge, England Markow, M. A., Institute of Nuclear Physics, Moscow, U.S.S.R.

Marx, G., Etvös Fizikai Társulat, Budapest, Hungary

Meader, D., Université de Genève, Genève, Switzerland Melvin, M. A., Temple University, Philadelphia, U.S.A.

Misner, C. W., University of Maryland, College Park, U.S.A.

Mфller, C., NORDITA, K $\phi$ benhavn, Denmark

Nadiozhan, P. K., Institute of Applied Mathematics, Moscow, U.S.S.R.

Newman, E. T., University of Pittsburgh, Pittsburgh, U.S.A.

Novikov, I. D., Institute of Applied Mathematics, Moscow, U.S.S.R.

Nutku, Y., Middle Eastern Technical University, Ankara, Turkey

Ozernoy, L. M., Lebedev Institute of Physics, Moscow, U.S.S.R.

Paal, G., Konkoly Observatory, Budapest, Hungary

Paczyński, B., Zakład Astronomii PAN, Warszawa, Poland 
Partridge, B., Haverford College, Haverford, U.S.A.

Pathria, R. K., University of Waterloo, Waterloo, Canada

Penrose, R., Oxford University, Oxford, England

Persides, S., University of Thessaloniki, Thessaloniki, Greece

Piotrowski, S., Zakład Astronomii PAN, Warszawa, Poland

Platania, G., Osservatorio Astronomico Capodimonte, Napoli, Italy

Plebański, J. F., Uniwersytet Warszawski, Warszawa, Poland

Poveda, A., Universidad National Autonoma, Mexico City, Mexico

Press, W. H., California Institute of Technology, Pasadena, U.S.A.

Raine, D., University Observatory, Oxford, England

Rees, M., University of Sussex, Brighton, England

Roeder, R. C., University of Toronto, West Hill, Canada

Rosenblum, A., Universität Bonn, Bonn, F.R.G.

Roxburgh, I. W., Queen Mary College, London, England

Ruffini, R., Princeton University, Princeton, U.S.A.

de Sabbata, V., Bologna University, Bologna, Italy

Sato, H., Kyoto University, Kyoto, Japan

Schmutzer, E., Friedrich-Schiller Universität, Jena, G.D.R.

Schramm, D., University of Texas, Austin, U.S.A.

Sedrakian, D. M., Erevan State University, Erevan, U.S.S.R.

Sexl, R., Universität Wien, Wien, Austria

Shvartsman, V. F., Special Astrophysical Observatory, St. Zelenchukskaya, U.S.S.R.

Sida, D. W., Carleton University, Ottawa, Canada

Silk, J., University of California, Berkeley, U.S.A.

Smak, J., Zakład Astronomii PAN, Warszawa, Poland

Sokolov, A. A., Moscow State University, Moscow, U.S.S.R.

Solheim, J. E., Auroral Observatory, Troms $\phi$, Norway

Spyrou, N., University of Thessaloniki, Thessaloniki, Greece

Starobinsky, A. A., Landau Institute for Theoretical Physics, Moscow, U.S.S.R.

Stephani, H., Universitaet Jena, D.D.R.

Stewart, J. M., Max-Planck-Institut, München, F.R.G.

Sugimoto, D., University of Tokyo, Tokyo, Japan

Teitelboim, C., Princeton University, Princeton, U.S.A.

Teukolsky, S., California Institute of Technology, Pasadena, U.S.A.

Thorne, K. S., California Institute of Technology, Pasadena, U.S.A.

Tiomno, J., Institute of Advanced Study, Princeton, U.S.A.

Trautman, A., Uniwersytet Warszawski, Warszawa, Poland

Tsuruta, S., NASA, Greenbelt, U.S.A.

Tyson, J. A., Bell Laboratoires, Murray Hill, U.S.A.

Urbantke, M., Universität Wien, Wien, Austria

Vilain, C., Observatoire de Paris, Meudon, France

Wagoner, R. V., Cornell University, Ithaca, U.S.A.

Walker, M., Max-Planck-Institut, München, F.R.G. 
Weber, J., University of Maryland, College Park, U.S.A.

Westervelt, P. J., University of Texas, Austin and Brown University, Providence, U.S.A.

Wheeler, J. A., Princeton University, Princeton, U.S.A.

Witten, L., University of Cincinnati, Cincinnati, U.S.A.

Woodhouse, N. J. M., King's College, London, England

Zel'dovich, Ya. B., Institute of Applied Mathematics, Moscow, U.S.S.R 